\title{
A Direct Method of Designing Filter Dividers With In Phase or Out of Phase Outputs
}

\author{
Xing-Jian Zhong ${ }^{1,}$, Kun Li, Zheng Chen, De-Xin Qu, Ying-Song ZhangSan \\ ${ }^{1}$ College of Communications Engineering, PLA University of Science \& Technology, Nanjing 210007 , \\ China.
}

a2052995300@qq.com

Keywords: Filter divider, coupling matrix, out of phase.

Abstract. A direct method of designing filter dividers is presented in this paper. The method is based on the filter divider's coupling matrix derived from coupling matrix of the associated filter. Through properly placing the output resonators, in phase or out of phase outputs could be obtained. Examples are given, the measured results accord well with the simulated results, showing validity of the method.

\section{Introduction}

Microwave dividers are widely used in so many cases such as antenna arrays, power systems, etc. Many works have been done about microwave dividers. Usually used microwave divider is Wilkinson divider which uses impedance transmission lines to ensure that characteristic impedance of each port is the same one, usually $50 \Omega$ [1-2]. Since divider is a circuit with 3 ports, it could also be designed using multiple network theory [3-4]. If special frequency selectivity is needed with a divider, the divider could be designed starting from a filter [5-8]. The work [5] gives an example. However, optimization is used in the design process. In this paper, a direct method without any optimization is proposed to design filter dividers based on coupling matrix derived from coupling matrix of the associated filter. Based on this method, the design of a filter divider is just like the design of a filter except some parameter managements. Through properly placing the resonators at the output ports, the filter dividers designed could easily have in phase or out of phase output signals.

\section{Organization of the Text}

Design Method of the Filter Divider. Fig. 1 shows a schematic of a third order bandpass filter, where denotes the ports, denotes the resonators, and denotes the coupling between the resonators or the resonator and the port, the associated coupling matrix is shown in (1).

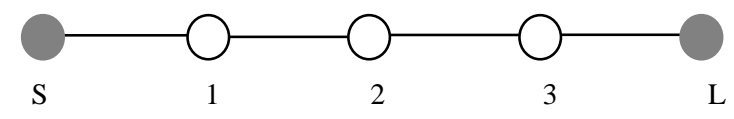

Fig. 1 Schematic of a bandpass filter of order three

$$
[m]=\left[\begin{array}{ccccc}
m_{S S} & m_{S 1} & 0 & 0 & 0 \\
m_{S 1} & m_{11} & m_{12} & 0 & 0 \\
0 & m_{12} & m_{22} & m_{23} & 0 \\
0 & 0 & m_{23} & m_{33} & m_{3 L} \\
0 & 0 & 0 & m_{3 L} & m_{L L}
\end{array}\right]
$$

From the proposed filter in Fig. 1, a filter divider could be derived like the one presented in Fig. 2, where the divider has two output ports. The coupling matrix of the filter divider is shown in (2). 


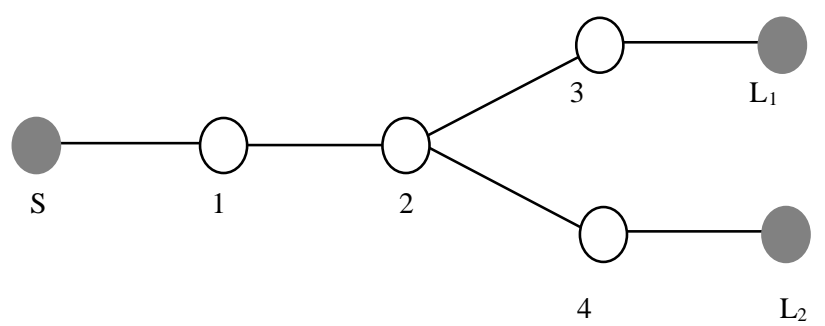

Fig. 2 Schematic of the filter divider derived from Fig. 1

$$
[m 1]=\left[\begin{array}{ccccccc}
\mathrm{m}_{\mathrm{sS}}^{\prime} & m_{S 1}^{\prime} & 0 & 0 & 0 & 0 & 0 \\
m_{S 1}^{\prime} & m_{11}^{\prime} & m_{12}^{\prime} & 0 & 0 & 0 & 0 \\
0 & m_{12}^{\prime} & m_{22}^{\prime} & m_{23}^{\prime} & m_{24}^{\prime} & 0 & 0 \\
0 & 0 & m_{23}^{\prime} & m_{33}^{\prime} & 0 & m_{3 L_{1}}^{\prime} & 0 \\
0 & 0 & m_{24}^{\prime} & 0 & m_{44}^{\prime} & 0 & m_{4 L_{2}}^{\prime} \\
0 & 0 & 0 & m_{3 L_{1}}^{\prime} & 0 & m_{L_{1} L_{1}}^{\prime} & 0 \\
0 & 0 & 0 & 0 & m_{4 L_{2}}^{\prime} & 0 & m_{L_{2} L_{2}}^{\prime}
\end{array}\right]
$$

The main work is to obtain coupling matrix $\mathrm{m} 1$ from coupling matrix $\mathrm{m}$. Since the filter divider derived from the filter, the parameters before the second resonator could be left unchanged, that means $m_{\mathrm{SS}}^{\prime}=m_{S S}, m_{11}^{\prime}=m_{11}, m_{22}^{\prime}=m_{22}, m_{12}^{\prime}=m_{12}$, and $m_{S 1}^{\prime}=m_{S 1}$. The next step is to obtain $m_{23}^{\prime}, m_{24}^{\prime}$, $m_{3 L_{1}}^{\prime}$ and $m_{4 L_{2}}^{\prime}$. It is known that the coupling coefficient $m_{23}$ denotes the coupling between resonator 2 and resonator 3 in Fig. 1. It also determines the power transmitted from resonator 2 to resonator 3 . To let the filter divider have selectivity performance as the original filter, the total power transmitted from resonator 2 to resonator 3 and resonator 4 in Fig. 2 should equal to the power transmitted from resonator 2 to resonator 3 in Fig. 1. According to this point, the relation below could be obtained,

$$
\left|\mathrm{m}_{23}^{\prime}\right|=\left|\mathrm{m}_{24}^{\prime}\right|=\left|\mathrm{m}_{23}\right| / \sqrt{2}
$$

Similarly, the output power from port L1 or port L2 in Fig. 2 should be same with the power output from port L in Fig.1 just with a coefficient of 1/2. Since the power accepted by resonator 3 or resonator 4 from resonator 2 in Fig. 2 is already half of the power accepted by resonator 3 from resonator 2 in Fig. 1, the parameters of each output branch in Fig. 2 should be same with the parameters in Fig. 1, thus the following formulas could be obtained,

$$
\begin{aligned}
& \mathrm{m}_{33}^{\prime}=\mathrm{m}_{44}^{\prime}=m_{33} \\
& \mathrm{~m}_{3 \mathrm{~L}_{1}}^{\prime}=\mathrm{m}_{4 L_{2}}^{\prime}=m_{3 L} \\
& m_{L_{L_{1}} L_{1}}^{\prime}=m_{L_{2} L_{2}}^{\prime}=m_{L L}
\end{aligned}
$$

Now the coupling matrix of the filter divider is totally determined. To verify the validity of the obtained coupling matrix, an example is given with parameters shown below [6-8],

Center frequency: $3000 \mathrm{MHz}$

Bandwidth: $\quad 120 \mathrm{MHz}$

$$
[m]=\left[\begin{array}{ccccc}
0 & 1.11 & 0 & 0 & 0 \\
1.11 & 0 & 1.064 & 0 & 0 \\
0 & 1.064 & 0 & 1.064 & 0 \\
0 & 0 & 1.064 & 0 & 1.11 \\
0 & 0 & 0 & 1.11 & 0
\end{array}\right]
$$

The simulated responses of the filter are given in Fig. 3. 


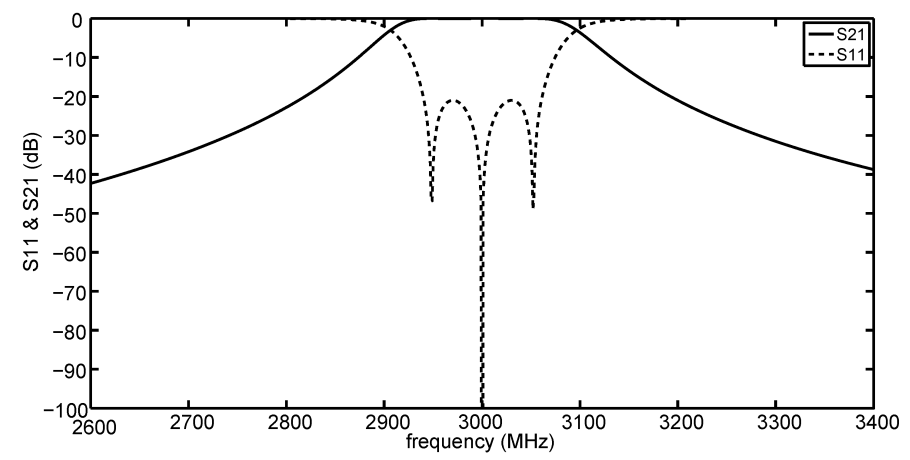

Fig. 3 Simulated responses of the filter

Based on the filter coupling matrix, using formulas above, the coupling matrix of the filter divider could be obtained as formula (6).

$$
[m 1]=\left[\begin{array}{ccccccc}
0 & m_{S 1}^{\prime} & 0 & 0 & 0 & 0 & 0 \\
m_{S 1}^{\prime} & 0 & m_{12}^{\prime} & 0 & 0 & 0 & 0 \\
0 & m_{12}^{\prime} & 0 & m_{23}^{\prime} & m_{24}^{\prime} & 0 & 0 \\
0 & 0 & m_{23}^{\prime} & 0 & 0 & m_{3 L_{1}}^{\prime} & 0 \\
0 & 0 & m_{24}^{\prime} & 0 & 0 & 0 & m_{4 L_{2}}^{\prime} \\
0 & 0 & 0 & m_{3 L_{1}}^{\prime} & 0 & 0 & 0 \\
0 & 0 & 0 & 0 & m_{4 L_{2}}^{\prime} & 0 & 0
\end{array}\right]
$$

where

$$
\begin{aligned}
& m_{S 1}^{\prime}=m_{3 L_{1}}^{\prime}=m_{4 L_{2}}^{\prime}=1.11 \\
& m_{12}^{\prime}=\sqrt{2} m_{23}^{\prime}=\sqrt{2} m_{24}^{\prime}=1.064
\end{aligned}
$$

Fig. 4 gives the simulated responses of the filter divider. It could be seen that the S11 in Fig. 3 is identical to the S11 in Fig. 4. The S21 and S31 in fig. 4 are identical, and also identical to the S21 in Fig. 3 with only $3 \mathrm{~dB}$ lower. So the frequency selectivity performance of the filter is held by the filter divider.

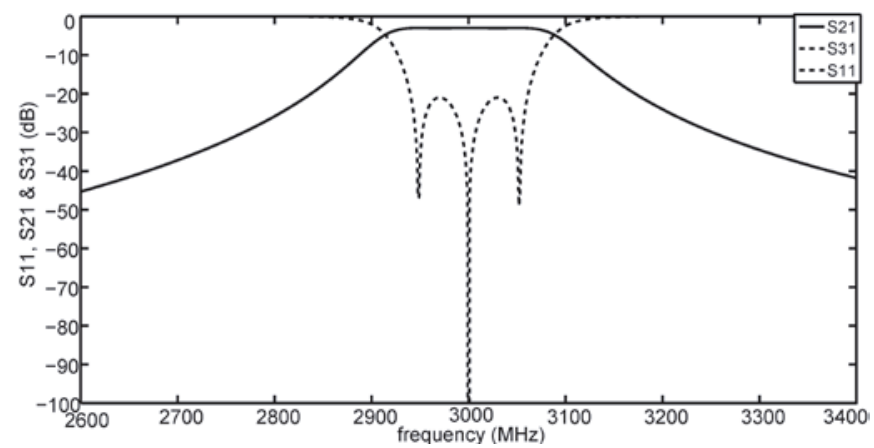

Fig. 4 Simulated responses of the divider

In the above example, the filter divider would have output signals with same phases since $m_{23}^{\prime}=m_{24}^{\prime}$. If having $m_{23}^{\prime}=-m_{24}^{\prime}$, the phases of the output signals would be opposite. The signs of the coupling coefficients could be determined by properly placing the output resonators. Fig. 5 gives an example.

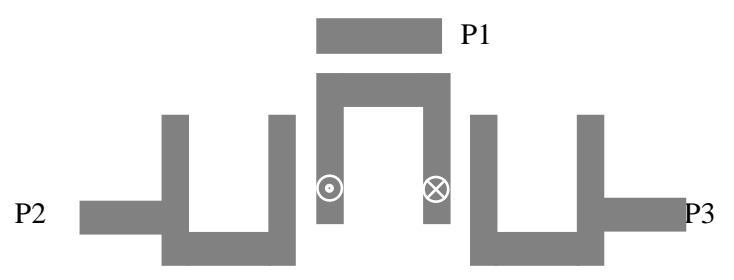

Fig. 5 A filter divider with output signals out of phase 
In Fig. 5, P1 is the input port, P2 and P3 are the output ports which are symmetrically placed. It could be clearly seen that the electric fields in one half of the resonator would be opposite to the electric fields in the other half. Thus the exciting electric fields of the output resonators are opposite, so P2 and P3 would have opposite output phases.

Design Examples and Measurements. According to the coupling matrix in (6), two microstrip filter dividers are designed, fabricated and measured. The thick of the used dielectric layer is $0.508 \mathrm{~mm}$ and the relative permittivity is 2.2. The measurement instrument is Agilent PNA 5230C.

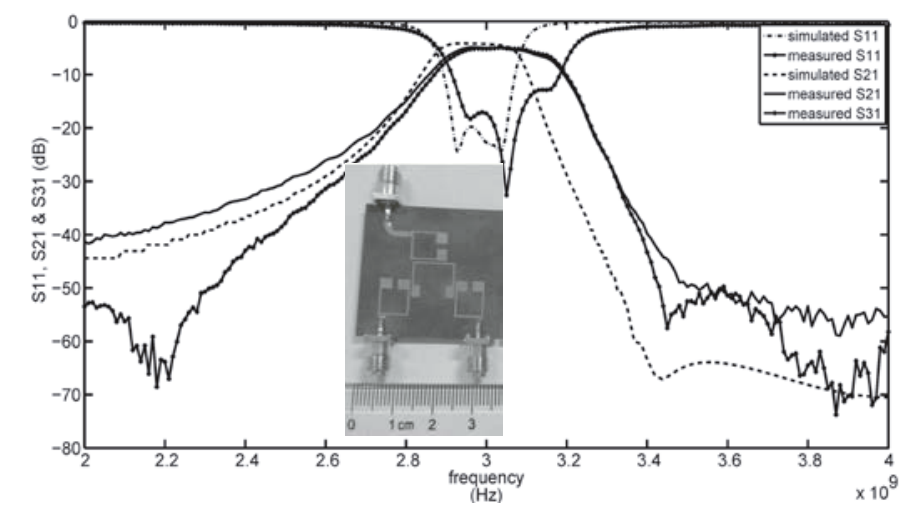

Fig. 6 Simulated and measured results of the filter divider with output signals out of phase (photo of the divider is embedded)

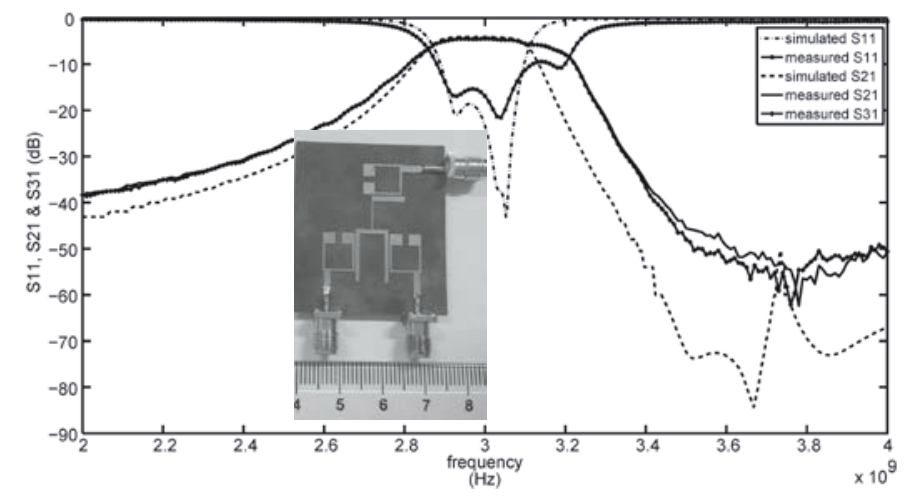

Fig. 7 Simulated and measured results of the filter divider with output signals in phase (photo of the divider is embedded)

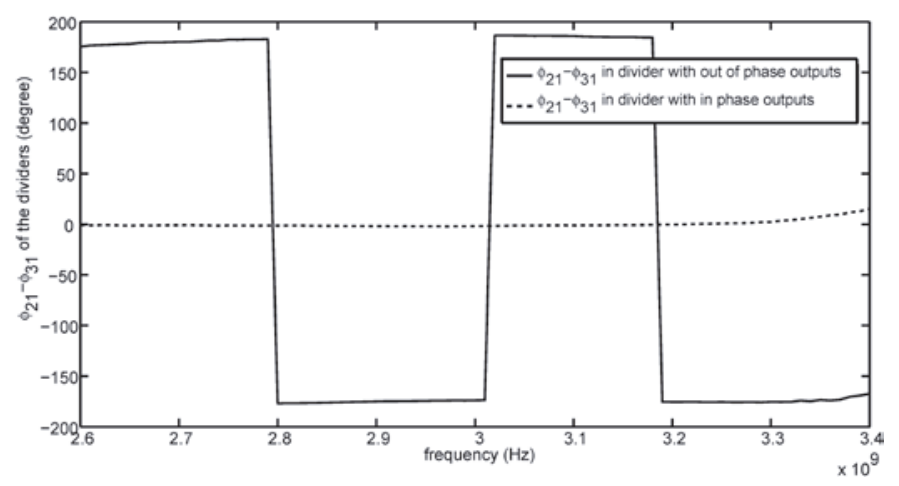

Fig. 8 Output phase differences of the dividers

The results above show that in both dividers the $\left|S_{21}\right|$ of each two output ports are slightly different in the signal band, the maximum differences between the two output ports are less than $2.1 \mathrm{~dB}$ in the band from $2.6 \mathrm{GHz}$ to $3.4 \mathrm{GHz}$. Because of the tapping feed lines, transmission zeros could be seen above the signal band. These transmission zeros enhance the signal suppression in the upper stopband. For the divider with out of phase outputs, the resonator which is between the two output resonators introduces other transmission zeros in the lower stopband for each signal path. These transmission zeros improve the performance in the lower stopband as shown in Fig. 6. A larger measured 
bandwidth could also be seen in the figures as compared to the simulated ones. This may be caused by the difference between the relative permittivity used in the simulation and the real relative permittivity of the dielectric layers. The output phase difference of the divider with in phase outputs is less than 1 degree, and the phase difference of the divider with out of phase outputs is 180 degree with deviation less than 8 degree from $2.6 \mathrm{GHz}$ to $3.4 \mathrm{GHz}$.

\section{Summary}

This paper gives a direct method to design filter dividers with in phase or out of phase outputs. This method makes the design of filter dividers like the design of a filter. And the in phase or out of phase outputs needed could be obtained just by properly placing the output resonators. Examples are given, the measured results accord well with the simulated results, showing validity of the proposed method.

\section{Acknowledgments}

This work was supported by the National Natural Science Foundation of China (NSFC) under Grant no. 61271105.

\section{References}

[1] K. H. Yi and B. K. Kang, "Modified Wilkinson Power Divider for nth Harmonic Suppression," IEEE Microwave and Wireless Components Letters, no. 5, vol. 13(2003) 178-180.

[2] E. Wilkinson, “An N-way hybrid power divider,” IRE Trans. Microwave Theory Tech., vol. 8(1960) 116-118.

[3] A. G. Lamperez, M. S. Palma and T. K. Sarkar, "Analytical synthesis of microwave multiport networks,” IEEE MTT-S Int. Microw. Symp. Dig., (2004)455-458.

[4] G. Macchiarella and S. Tamiazzo, “ Novel Approach to the Synthesis of Microwave Diplexers,’IEEE Trans. Microw. Theory Tech., no. 12, vol. 54(2006) 4281-4290.

[5] M. Guglielmi, B. Gimeno and T. Kjoede, “ A new equiripple power splitter for radiolink applications,”28th European Microwave conference, (1998) 30-32

[6] J. S. Hong and M. J. Lancaster, Microstrip Filters for RF/Microwave Applications. New York: Wiley, 2001.

[7] R. J. Cameron, "General coupling matrix synthesis methods for Chebyshev filtering functions," IEEE Trans. Microw. Theory Tech., vol. 47(1999) 433-442.

[8] A. E. Atia, A. E. Williams and R. W. Newcomb, “ Narrow-band multiple-coupled cavity synthesis,” IEEE Trans. Circuit syst., vol. 21(1974) 649-655. 